\title{
Greater cardiomyocyte density on aligned compared with random carbon nanofibers in polymer composites
}

This article was published in the following Dove Press journal:

International Journal of Nanomedicine

28 November 2014

Number of times this article has been viewed

\author{
Abdullah M Asiri' \\ Hadi M Marwani' \\ Sher Bahadar Khan' \\ Thomas J Webster ${ }^{1,2}$ \\ 'Center of Excellence for Advanced \\ Materials Research, King Abdulaziz \\ University, Jeddah, Saudi Arabia; \\ ${ }^{2}$ Department of Chemical \\ Engineering, Northeastern University, \\ Boston, MA, USA
}

\begin{abstract}
Carbon nanofibers (CNFs) randomly embedded in poly(lactic-co-glycolic-acid) (PLGA) composites have recently been shown to promote cardiomyocyte growth when compared with conventional PLGA without CNFs. It was shown then that PLGA:CNF composites were conductive and that conductivity increased as greater amounts of CNFs were added to pure PLGA. Moreover, tensile tests showed that addition of CNFs increased the tensile strength of the PLGA composite to mimic that of natural heart tissue. Most importantly, throughout all cytocompatibility experiments, cardiomyocytes were viable and expressed important biomarkers that were greatest on 50:50 wt \% CNF:PLGA composites. The increased selective adsorption of fibronectin and vitronectin (critical proteins that mediate cardiomyocyte function) onto such composites proved to be the mechanism of action. However, the natural myocardium is anisotropic in terms of mechanical and electrical properties, which was not emulated in these prior PLGA:CNF composites. Thus, the aim of this in vitro study was to create and characterize CNFs aligned in PLGA composites (at 50:50 wt\%, including their mechanical and electrical properties and cardiomyocyte density), comparing such results with randomly oriented CNFs in PLGA. Specifically, CNFs were added to soluble biodegradable PLGA (50:50 PGA:PLA weight ratio) and aligned by applying a voltage and then allowing the polymer to cure. CNF surface micron patterns ( $20 \mu \mathrm{m}$ wide) on PLGA were then fabricated through a mold method to further mimic myocardium anisotropy. The results demonstrated anisotropic mechanical and electrical properties and significantly improved cardiomyocyte density for up to 5 days on CNFs aligned in PLGA compared with being randomly oriented in PLGA. These results indicate that CNFs aligned in PLGA should be further explored for improving cardiomyocyte density, which is necessary in numerous cardiovascular applications.
\end{abstract}

Keywords: cardiomyocytes, poly(lactic-co-glycolic acid), carbon nanofibers, aligned, nanotechnology, anisotropy

\section{Introduction}

Nanotechnology has started to revolutionize medical devices. ${ }^{1}$ While there has been a significant number of studies examining the role of nanotechnology in improving orthopedic, vascular, cartilage, and nervous system applications, the heart has been largely ignored to date. ${ }^{1}$ Nanomaterials are intriguing materials for regenerating cardiovascular tissue since they can mimic natural proteins (such as collagen) in the heart, so can mimic numerous properties of natural heart tissue (such as cytocompatibility, along with mechanical and electrical properties). Moreover, small changes in nanoscale surface feature sizes and geometries on the surface of biomaterials can significantly alter surface energetics to increase tissue growth, inhibit infection, or decrease inflammation. ${ }^{2}$
Correspondence: Thomas J Webster Department of Chemical Engineering, Northeastern University, 3 I 3 Snell Engineering Building, 360 Huntington Avenue, Boston, MA 02115, USA

Tel +l 6173836585

Fax + I 6173836586

Email th_webster@neu.edu 
One particular nanomaterial that has been initially studied for its cardiovascular applications is a poly(lacticco-glycolic acid) (PLGA) composite containing embedded carbon nanofibers (CNFs) to create a "patch" for an infarcted area of the heart. ${ }^{3}$ The idea here is that once heart damage has occurred through a heart attack or any other event, a material can be injected or sutured on top of the damaged tissue to rapidly regenerate healthy heart tissue in its place. Recent research has shown when one adds CNFs to PLGA, cardiomyocytes (specific heart tissue cells responsible for contraction) will adhere and proliferate to a greater extent than on their non-nano-reinforced counterparts. ${ }^{3}$ It was demonstrated in such earlier work that PLGA:CNF materials were conductive, and that their conductivity increased as increasing amounts of CNFs were added to pure PLGA. Specifically, conductivity increased from $0 \mathrm{~S} \cdot \mathrm{m}^{-1}$ for pure PLGA $(100: 0 \mathrm{wt} \%)$ to $5.5 \times 10^{-3} \mathrm{~S} \cdot \mathrm{m}^{-1}$ for pure CNFs $(0: 100 \mathrm{wt} \%) .{ }^{3} \mathrm{CNFs}$ are a less expensive alternative of carbon nanotubes, but are still highly conductive, strong, and mimic the natural dimensions of collagen. CNFs have even been added to self-assembled materials (like helical rosette nanotubes) to develop a conductive material that could be injected into or onto damaged heart tissue as a liquid which can then solidify to a viscous gel to heal damaged heart tissue. ${ }^{4}$

Tensile tests showed that addition of CNFs increased the tensile strength of the PLGA composite to mimic that of natural heart tissue (from 0.15 MPa for 100\% PLGA to $5.41 \mathrm{MPa}$ for the 50:50 [PLGA:CNF $\{\mathrm{wt} \%$ : $\mathrm{wt} \%\}$ ] ratio at $0.025 \mathrm{~g} / \mathrm{mL}) .{ }^{5}$ Most importantly, the results indicated that throughout all cytocompatibility experiments, cardiomyocytes were viable and expressed important biomarkers (such as troponin-T, connexin-42, and alpha-sarcomeric actin) and that cardiomyocyte density was the highest on PLGA:CNF composites at a 50:50 (wt \%) ratio (a 55\% increase in cardiomyocyte density after 120 hours compared with pure PLGA and a $75 \%$ increase compared with the control at the same time point). ${ }^{5}$ This is in contrast with other research suggesting that CNFs are highly toxic. ${ }^{1}$

However, in all of these previous studies, CNFs were randomly oriented into PLGA scaffolds, whereas natural cardiac tissue possesses aligned nanostructured features. For example, natural heart tissue is quite anisotropic, ranging from $0.16 \mathrm{~S} \cdot \mathrm{m}^{-1}$ longitudinally to $0.005 \mathrm{~S} \cdot \mathrm{m}^{-1}$ transversely. It is hypothesized that cardiomyocyte function can be further enhanced on composites of CNFs aligned in PLGA since the electrical and mechanical properties of such composites will be more similar to those of the natural myocardium. Thus, the purpose of this innovative in vitro study was to create anisotropic CNFs in a PLGA composite and determine their potential to serve as an improved cardiac patch.

\section{Materials and methods Fabrication of PLGA:CNF}

PLGA:CNF composites were fabricated as described in considerable detail in our previous studies. ${ }^{3,5}$ Since the 50:50 $\mathrm{wt} \%$ CNF in PLGA composites in our earlier studies demonstrated the best cardiomyocyte function, this weight percentage was selected here. PLGA composites $(0.025 \mathrm{~g} / \mathrm{mL}$; 50:50 PLA:PGA wt\%; Polysciences, Warrington, PA, USA; \#23986) were created by first diluting tetrahydrofuran (Mallinckrodt Chemicals, Phillipsburg, NJ, USA; \#C45763) and then sonicating the PLGA pellets in tetrahydrofuran in a water bath (B3500A-DTH; VWR, Radnor, PA, USA) below $30^{\circ} \mathrm{C}$ for 30 minutes. Next, $500 \mathrm{mg}$ of CNFs $(99.9 \%$ by wt $\%$, unfunctionalized; Catalytic Materials, Pittsboro, NC, USA) with a diameter of $100 \mathrm{~nm}$ and length of $100 \mu \mathrm{m}$ were sonicated (Sonicator 3000; Misonix, Farmingdale, NY, USA) in chloroform (Fisher Scientific, Waltham, MA, USA; \#102591) at $20 \mathrm{~W}$ for 30 minutes. After obtaining the separately sonicated PLGA and CNF solutions, various PLGA:CNF solutions at $50: 50 \mathrm{wt} \%$ ratios were fabricated by adding the appropriate amount of CNF to PLGA. The CNF weight ratios were measured using a laboratory balance (AL54; Mettler Toledo, Columbus, OH, USA). When the appropriate ratio was added, each composite material was sonicated (Sonicator 3000; Misonix) at $10 \mathrm{~W}$ for 20 minutes each.

Next, a $22 \mathrm{~mm}$ diameter microscope cover glass (Circles No 1, 0.13-0.17 mm thick; size 22 mm; \#12-545-101; Fisher Scientific) was coated with the aforementioned composite. First, the glass substrate was cleaned by soaking in a 70:30 (volume \%) ethanol-deionized solution while shaking (Advanced Digital Shaker; VWR) for 10 minutes. Next, the substrate was added to a $100 \%$ deionized water solution and shaken for 10 minutes. Using a disposable pipette (Fisherbrand, Waltham, MA, USA; \#13-711-9 AM), 1 mL of the PLGA:CNF composite solution was placed onto the glass substrate and put into an oven at $42^{\circ} \mathrm{C}$ for 15 minutes to cure. Each composite film was then vacuum-dried using Vacuum Oven 3500 (Shel Lab, Cornelius, OR, USA) at 20 inches of mercury gauge vacuum pressure for 48 hours to allow the tetrahydrofuran and chloroform to evaporate. Similar experiments were conducted for controls (no CNFs). All the samples were sterilized using ultraviolet light for 24 hours prior to cell experiments.

To create aligned CNFs in PLGA, a similar procedure to that described above was followed, except that the 
PLGA:CNF composites in solution were exposed to $20 \mathrm{~V}$ for 24 hours after being placed on a coverslip while being allowed to cure. Special care was taken to minimize an increase in temperature that occurred while applying the voltage by placing the Petri dish containing the PLGA:CNF composites in ice for the 24 hours. Moreover, after solidification, micron patterns of CNFs were created on the PLGA by placing gold-coated grids with grooves (widths of $20 \mu \mathrm{m}$; SPI Supplies, West Chester, PA, USA; \#2422G-XA) on top of the PLGA coating on the coverslips. The CNF-containing PLGA solution was then placed into the grooves of the gold grid using a micropipette. The grid with embedded CNFs on top of the PLGA-coated glass was then placed in a vacuum oven for 48 hours, after which the gold grid was removed, the substrate was rinsed with deionized water, and microscale patterns of CNFs on PLGA were created.

\section{Material characterization}

\section{Scanning electron microscopy}

A scanning electron microscope (model 2700; Hitachi, Tokyo, Japan) was used to characterize the surface of the PLGA:CNF samples.

\section{Atomic force microscopy}

Atomic force microscopy measurements were performed using a scanning probe microscope (MFP-3D Coax; Asylum Research, Santa Barbara, CA, USA) to create a topographical map of the surface. The analyses were performed in air and under a constant applied force condition (non-contact mode) with a cantilever (Asylum Research; \#AC160TS) resonant frequency of $\sim 360 \mathrm{kHz}$ and a theoretical spring constant of $\mathrm{k}$ : $42 \mathrm{~N} \cdot \mathrm{m}^{-1}$. Images were processed and analyzed using Image Processing Data Analysis Igor Pro 6 software (WaveMetrics, Tigard, OR, USA).

\section{Conductivity tests}

Conductivity tests were performed to compare the characteristics of the study material with values for the human myocardium reported in the literature (Table 1). The electrical resistance of the dry PLGA:CNF samples were obtained by measuring sample resistance in the horizontal and vertical directions to assess anisotropy. A four-point probe measurement was completed with one pair of leads used to inject the measurement current, while a second pair of leads, in parallel with the first, was used to measure the potential drop across the device. There was no potential drop across the voltage measurement leads, so the contact resistance drop was not included, making a more accurate system. All measurements were conducted using a four-point conductivity measurement device built in-house, and were completed four times on each sample at different places on the samples.

\section{Tensile tests}

Tensile tests were performed to compare the characteristics of the study material with those reported in the literature for human myocardium. A flat test sample mold made from Sylgard $184^{\circledR}$ (Dow Corning, New York, NY, USA; \#11-1792-01) with a gauge length of $7.62 \mathrm{~mm}$, width of gauge length of $3.18 \mathrm{~mm}$, reduced section of $9.53 \mathrm{~mm}$, distance between shoulders of $26.33 \mathrm{~mm}$, overall length of $63.55 \mathrm{~mm}$, width of grip section of $9.53 \mathrm{~mm}$, and a sample thickness of $3.20 \mathrm{~mm}$ was used to create the tensile samples. The PLGA:CNF composite was added to the mold and vacuum-dried (Shel Lab) at 20 inches of mercury gauge vacuum pressure for 72 hours to allow for solvent evaporation. In the case of the anisotropic samples, the same methods as used above were followed (ie, applying a voltage and creating micron patterns of CNFs on the PLGA). After the samples were dried and released from the mold, tensile tests were performed in a tensile machine (Minimat 2000; Rheometric Scientific Inc, Piscataway, NJ, USA) at a speed of $50 \mathrm{~mm}$ per minute until material failure occurred in accordance with the American Society for Testing and Materials Standard Test Method for Tensile Properties of Plastics Designation D638-10. Experiments were completed in quadruplicate per composite.

\section{Cardiomyocyte adhesion and proliferation density}

Lastly, for the analysis of cell adhesion and proliferation in vitro, human cardiomyocytes (Celprogen Inc, Torrance, CA, USA; \#36044-15) were seeded at a cell density of 3,500 cells $/ \mathrm{cm}^{2}$ on all of the composites in complete growth medium (Celprogen Inc; \#M36044-15S) supplemented with $10 \%$ fetal bovine serum (Thermo Fisher Scientific, Waltham, MA, USA; \#SH30109) and a 1\% penicillin/streptomycin mixture (Thermo Fisher Scientific; \#BP2959-50). Cells were seeded into 12-well human cardiomyocyte stem cell culture extracellular matrix plates (Celprogen Inc; \#E36044-15-12 Well) on top of the PLGA:CNF composites. The samples were incubated for 4 hours for the cell adhesion assay and for 1,3 , and 5 days for the cell proliferation assay under standard incubation conditions $\left(5 \% \mathrm{CO}_{2}, 95 \%\right.$ humidified air, and $37^{\circ} \mathrm{C}$, changing the medium every other day). 3-(4,5-Dimethylthiazol-2-yl)-2,5-diphenyltetrazolium bromide (MTT; Promega, Madison, WI, USA; CellTiter $96^{\circledR}$ Non-Radioactive Cell Proliferation; \#G-4100) assays were undertaken 
according to the manufacturer's instructions to analyze the cytocompatibility of the composite. A standard curve was created to correlate absorbance with cell numbers.

\section{Statistical analysis}

All experiments were performed at least in triplicate with three repeats each. One-way analysis of variance and the Student's $t$-test were used to determine differences between means. A $P$-value of $<0.01$ was considered to be statistically significant.

\section{Results}

\section{Fabrication of materials}

Representative scanning electron microscopic images of the as-synthesized PLGA:CNF composite surfaces can be seen in Figure 1. The randomly oriented CNFs were uniformly dispersed within the PLGA matrix (Figure 1A). However, as expected, the aligned CNFs appeared in well-aligned $20 \mu \mathrm{m}$ patterns on the PLGA, and this was further confirmed by an atomic force microscopy line scan (Figure 1B and C).

\section{PLGA:CNF material properties}

\section{Conductivity}

The results of this study also demonstrate that the CNFs were aligned in PLGA since the vertical (or longitudinal) conductivity increased from 0.075 to $0.1 \mathrm{~S} / \mathrm{m}$ and the transverse (or horizontal) conductivity decreased from 0.075 to 0.0025 $\mathrm{S} / \mathrm{m}$ for the randomly oriented CNFs compared to those aligned in PLGA, respectively (Table 1). The conductivity of natural heart tissue ranges from $0.16 \mathrm{~S} / \mathrm{m}$ longitudinally (vertically) to $0.005 \mathrm{~S} / \mathrm{m}$ transversely (horizontally), ie, a factor of 32 which is more closely matched by the CNF aligned in PLGA composites fabricated here (a ratio of 40). ${ }^{5}$ Thus, the aligned CNFs in PLGA composites fabricated in the present study more accurately reflect the anisotropic properties
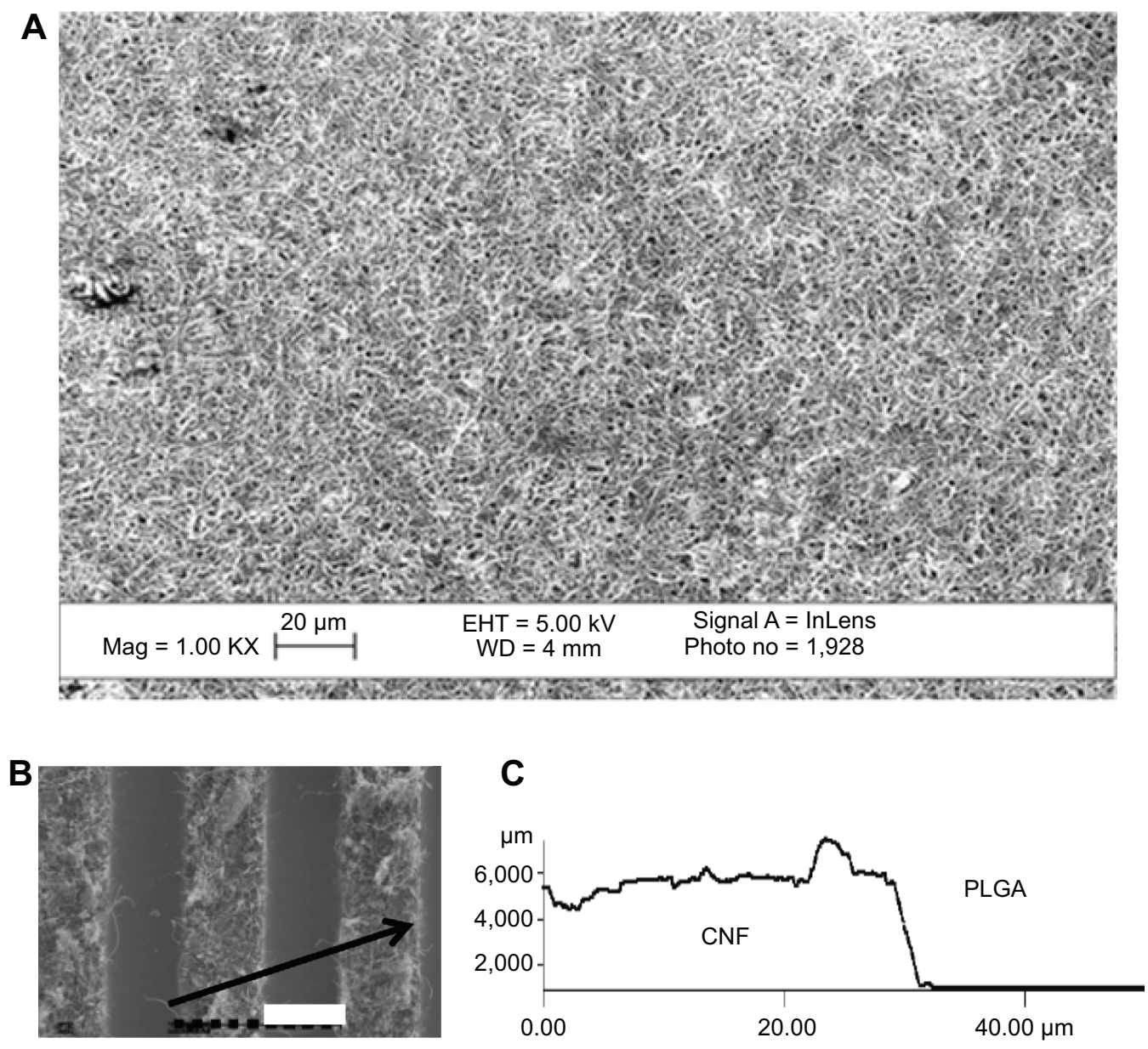

Figure I Scanning electron microscopic images showing the distribution of CNF in a PLGA matrix at 50:50 wt\%, with (A) random orientation and (B) aligned to mimic the natural anisotropy of cardiac tissue. The arrow is pointing to the atomic force microscope line scan of this region shown in $\mathbf{C}$. (C) Shows the atomic force microscopy line scan of the 50:50 CNF:PLGA aligned composite, demonstrating micron alignment of the CNFs on the PLGA surface. Scale bars (white bar) $20 \mu \mathrm{m}$.

Abbreviations: CNF, carbon nanofiber; EHT, extra high; Mag, magnification; no, number; PLGA, poly(lactic-co-glycolic-acid); WD, working distance; wt, weight. 
Table I Conductivity of materials of interest in the present study

\begin{tabular}{lll}
\hline Sample & Vertical conductivity (S/m) & Horizontal conductivity (S/m) \\
\hline Pure PLGA & 0 & 0 \\
50:50 wt\% CNFs randomly oriented in PLGA & $0.075 \pm 0.001$ & $0.075 \pm 0.001$ \\
$50: 50$ wt\% CNFs aligned in PLGA & $0.1 \pm 0.01$ & $0.0025 \pm 0.001$
\end{tabular}

Notes: The conductivity of natural heart tissue ranges from $0.16 \mathrm{~S} / \mathrm{m}$ longitudinally (vertically) to $0.005 \mathrm{~S} / \mathrm{m}$ transversely (horizontally) - a factor of 32 which is more closely matched by the CNFs aligned in PLGA composites fabricated here (a ratio of 40). Values are given as mean and standard deviation.

Abbreviations: CNFs, carbon nanofibers; PLGA, poly(lactic-co-glycolic-acid); S/m, Semens/meter; wt, weight.

of natural myocardium. As expected, without CNFs, pure PLGA was not conductive.

\section{Tensile tests}

Tensile tests also provided additional evidence of the alignment of CNFs in PLGA, with the results showing a Young's modulus of $5.05 \mathrm{mPa}$ compared with $7.90 \mathrm{MPa}$ in the vertical direction and 4.96 MPa compared with $2.14 \mathrm{MPa}$ in the horizontal direction for the randomly oriented CNFs versus aligned CNFs in PLGA, respectively (Table 2). Moreover, the elongation at break was measured to be $10.1 \%$ compared with $4.1 \%$ in the vertical direction and $11.3 \%$ compared with $14.8 \%$ in the horizontal direction for the randomly oriented CNFs versus the aligned CNFs in PLGA, respectively (Table 2). Compared with native cardiac heart tissue, the composites had mechanical properties that were stiffer than those in native heart muscle, which is made of collagen and is elastic with a stiffness of approximately $50-100 \mathrm{kPa}$ during diastole. ${ }^{6-9}$ However, the modulus of active myocardium during systole is approximately 20 -fold higher, ${ }^{10,11}$ which more closely resembles the modulus of the PLGA:CNF composites. As expected, all CNF-containing PLGA composites had mechanical properties that were closer to those of the natural myocardium when compared with pure PLGA.

\section{Cardiomyocyte density}

Lastly, the results of this study demonstrate for the first time that adhesion and proliferation of cardiomyocytes was greater on substrates that mimic the anisotropic electrical, and mechanical properties of the natural myocardium.
Specifically, after 4 hours of culture, the density of cardiomyocytes increased almost 1.5 times on aligned CNFs when compared with randomly oriented CNFs in PLGA (Figure 2). Moreover, the number of cardiomyocytes continued to increase up to 1.5 times on aligned CNFs when compared with randomly oriented CNFs in PLGA for up to 5 days in culture (Figure 3). Both types of CNF-containing PLGA composites showed greater cardiomyocyte numbers than pure PLGA with no CNFs.

\section{Discussion}

Numerous materials (such as alginate ${ }^{12}$ and polytetrafluoroethylene ${ }^{13}$ ) have been investigated for their ability to promote cardiomyocyte function after a myocardial infarction; however, most are not conductive and do not possess anisotropic conductive properties. Nonetheless, research has shown that some of these materials, in particular composite materials, can stabilize heart tissue and increase the regeneration of healthy cardiovascular tissue in the place of damaged heart tissue. One way to further improve materials for cardiac patch application is to use nanomaterials that can mimic the nanoarchitecture and anisotropy of natural heart tissue. One such example explored here is the use of a PLGA material with embedded CNFs. This present study continued this work as we developed aligned CNFs in PLGA (as opposed to randomly oriented CNFs in PLGA) to further mimic the properties of the myocardium.

It is intriguing to consider which properties of the aligned CNFs in PLGA are responsible for improved cardiomyocyte function. While this will require further

Table 2 Mechanical properties of the materials of interest in the present study

\begin{tabular}{|c|c|c|}
\hline Sample & $\begin{array}{l}\text { Vertical Young's modulus } \\
\text { (MPa } \pm \text { SEM) and elongation at } \\
\text { break }(\% \pm \text { SEM) }\end{array}$ & $\begin{array}{l}\text { Horizontal Young's modulus } \\
\text { (MPa } \pm \text { SEM) and elongation at break } \\
(\% \pm \text { SEM) }\end{array}$ \\
\hline Pure PLGA & $0.17 \pm 0.02$ and $|3| \pm I I . I$ & $0.15 \pm 0.01$ and $125 \pm 10.9$ \\
\hline 50:50 wt\% CNFs randomly oriented in PLGA & $5.05 \pm I . I$ and $10.1 \pm I .8$ & $4.96 \pm 1.3$ and $I I .3 \pm 2.1$ \\
\hline 50:50 wt\% CNFs aligned in PLGA & $7.90 \pm 1.8$ and $4.1 \pm 0.9$ & $2.14 \pm 0.3$ and $14.8 \pm 3.1$ \\
\hline
\end{tabular}

Abbreviations: CNFs, carbon nanofibers; PLGA, poly(lactic-co-glycolic-acid); SEM, standard error of the mean; wt, weight. 


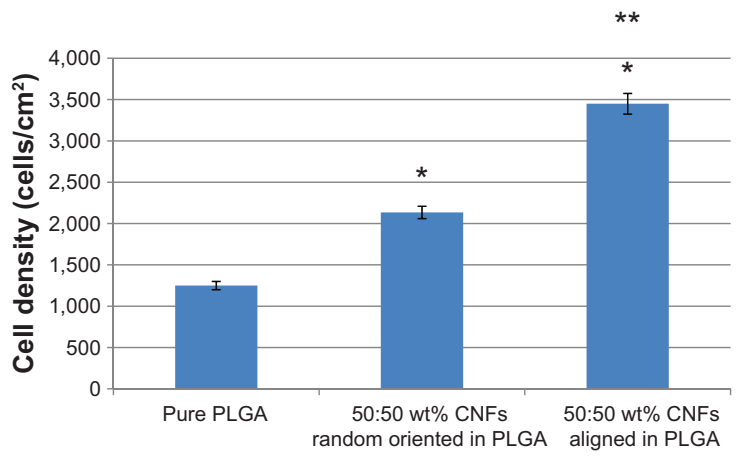

Figure 2 Increased cardiomyocyte adhesion on aligned CNFs compared with randomly oriented CNFs in PLGA composites.

Notes: The adhesion time was 4 hours. Seeding density 3,500 cells $/ \mathrm{cm}^{2}$. Data shown as the mean \pm standard error of the mean $(n=3)$. $* P<0.01$ compared with pure PLGA and $* * P<0.01$ compared with 50:50 wt\% CNFs randomly oriented in PLGA. Abbreviations: CNFs, carbon nanofibers; PLGA, poly(lactic-co-glycolic-acid); wt, weight.

investigation, it is known that conduction and mechanical properties alter cardiomyocyte function. The present results further demonstrate this by comparing each of the CNF:PLGA composites (aligned or not) with PLGA alone; cardiomyocyte density was improved on PLGA with conductive CNFs. However, what is not known at a cellular mechanistic level is how anisotropic scaffold properties (electrical or mechanical) alter cardiomyocyte function. For example, it is possible that such anisotropic properties would help cells align and form electrical or mechanical networks with each other, further helping cell-to-cell communications to aid in growth. It has also been demonstrated in other studies that proteins when initially adsorbing from plasma or serum, may adsorb to patterned CNFs to control cell functions such as alignment, geometry, and cell-to-cell contacts. ${ }^{14}$

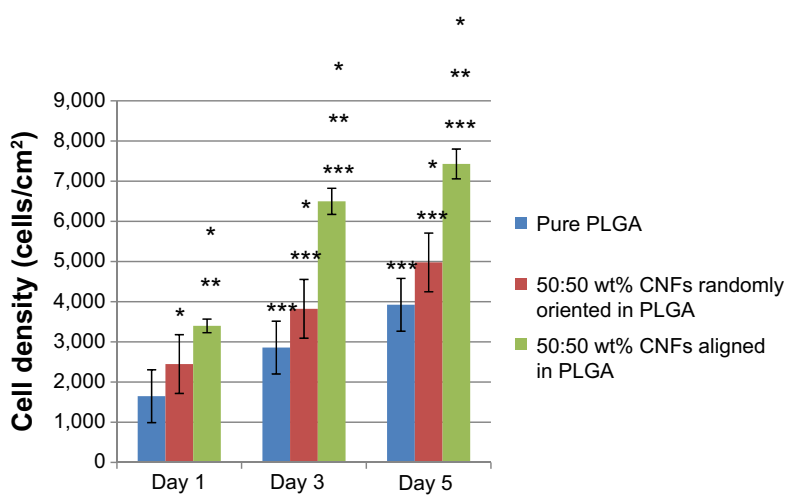

Figure 3 Increased cardiomyocyte growth on aligned CNFs compared with randomly oriented CNFs in PLGA composites.

Notes: Seeding density 3,500 cells $/ \mathrm{cm}^{2}$. Data shown as the mean \pm standard error of the mean $(n=3)$. $* P<0.01$ compared with pure PLGA at respective time period; $* * P<0.0$ I compared with 50:50 wt\% randomly oriented CNFs in PLGA at respective time period; $* * * P<0.01$ compared with previous time period, same sample.

Abbreviations: CNFs, carbon nanofibers; PLGA, poly(lactic-co-glycolic-acid); wt, weight.
Of course, a caution with the presently fabricated composites may be the potential toxicity of CNFs after the PLGA has degraded and new myocardial tissue has formed in the area. While many more studies need to be conducted, there is now a growing body of evidence suggesting that CNFs when made appropriately (ie, containing no impurities), have very low toxicity to many of the cells in the body, including osteoblasts, neurons, chondrocytes, smooth muscle cells, and according to the present data, cardiomyocytes..$^{15}$ Of course, future studies will need to explore other functions of cardiomyocytes, such as cardiac troponin- $\mathrm{T}$, connexin- 42 , and alpha-sarcomeric actin, which have a significant role in the function of cardiomyocytes.

Regardless of the reason, this study introduced a new material (CNFs aligned in PLGA) and a simple manner of producing such a material that should be studied further for cardiovascular applications. By controlling CNF alignment, weight percentage, and distribution in PLGA, it is clear that one can obtain a composite with properties that match those of the myocardium and are critical for improving the function of cardiomyocytes.

\section{Conclusion}

The aim of this in vitro study was to fabricate and characterize CNFs aligned in PLGA composites (at 50:50 wt\%) and compare the results (including mechanical, electrical, and cardiomyocyte function) with those obtained for randomly oriented CNFs in PLGA. Specifically, CNFs were added to soluble biodegradable PLGA (50:50 PGA:PLA wt\% ratio) and were aligned by applying a voltage and then allowing the polymers to cure. Surface micron patterns ( $20 \mu \mathrm{m}$ wide) for CNFs on PLGA were fabricated using a mold method. The results demonstrated anisotropic mechanical and electrical properties and significantly improved cardiomyocyte density for up to 5 days of culture on CNFs aligned in PLGA compared with CNFs that were randomly oriented. These results indicate that CNFs aligned in PLGA should be explored further for their ability to improve cardiomyocyte density, which is necessary in numerous cardiovascular applications.

\section{Acknowledgment}

This work was funded by the Deanship of Scientific Research (DSR), King Abdulaziz University, under grant number 25-130-35-HiCi. The authors, therefore, acknowledge technical and financial support from King Abdulaziz University.

\section{Disclosure}

The authors report no conflicts of interest in this work. 


\section{References}

1. Zhang L, Webster TJ. Nanotechnology and nanomaterials: promises for improved tissue regeneration. Nano Today. 2009;4(1):66-80.

2. Khang D, Kim SY, Liu-Synder P, Palmore GTR, Durbin SM, Webster TJ. Enhanced fibronectin adsorption on carbon nanotubes/poly(carbonate) urethane: independent role of surface nano roughness and associated surface energy. Biomaterials. 2007;28(32):4745-4768.

3. Stout DA, Basu B, Webster TJ. Poly(lactic-co-glycolic acid): carbon nanofiber composites for myocardial tissue engineering applications. Acta Biomater. 2011;7(8):3101-3112.

4. Meng X, Stout D, Sun L, Fenniri H, Webster TJ. Injectable biomimetic hydrogels with carbon nanofibers and novel self-assembled chemistries for myocardial applications. In: Narayan N, Bose S, Bandyopadhyay A, editors. Biomaterials Science: Processing, Properties and Applications II: Ceramic Transactions. Volume 237. Hoboken, NJ, USA: John Wiley and Sons, Inc; 2012.

5. Stout DA, Yoo J, Santiago-Miranda AN, Webster TJ. Mechanisms of greater cardiomyocyte functions on conductive nanoengineered composites for cardiovascular applications. Int J Nanomedicine. 2012;7: 5653-5669.

6. Christman KL, Lee RJ. Biomaterials for the treatment of myocardial infarction. J Am Coll Cardiol. 2006;48(5):907-913.

7. Engelmayr GC, Cheng M, Bettinger CJ, Borenstein JT, Langer R, Freed LE. Accordion-like honeycombs for tissue engineering of cardiac anisotropy. Nat Mater. 2008;7(12):1003-1010.
8. Jalil J, Doering C, Janicki J, Pick R, Shroff S, Weber K. Fibrillar collagen and myocardial stiffness in the intact hypertrophied rat left ventricle. Circ Res. 1989;64(6):1041-1050.

9. Mirsky I, Parmley WW. Assessment of passive elastic stiffness for isolated heart muscle and the intact heart. Circ Res. 1973;33(2):233-243.

10. Boublik J, Park H, Radisic M, et al. Mechanical properties and remodeling of hybrid cardiac constructs made from heart cells, fibrin, and biodegradable, elastomeric knitted fabric. Tissue Eng. 2005;11(7-8): 1122-1132.

11. Phillips CA, Petrofsky JS. Myocardial material mechanics: characteristic variation of the circumferential and longitudinal systolic moduli in left ventricular dysfunction. J Biomech. 1984;17(8):561-568.

12. Willems IE, Arends JW, Daemen MJ. Tenascin and fibronectin expression in healing human myocardial scars. J Pathol. 1996;179(3): 321-325.

13. Landa N, Miller L, Feinberg MS, et al. Effect of injectable alginate implant on cardiac remodeling and function after recent and old infarcts in rat. Circulation. 2008;117(11):1388-1396.

14. Bajaj P, Khang D, Webster TJ. Control of spatial cell attachment on carbon nanofiber patterns on polycarbonate urethane. Int J Nanomedicine. 2006;1(3):361-366.

15. Price RL, Webster TJ. Increased osteoblast viability in the presence of smaller nano-dimensioned carbon fibers. Nanotechnology. 2004;15(8): 892-900.
International Journal of Nanomedicine

\section{Publish your work in this journal}

The International Journal of Nanomedicine is an international, peerreviewed journal focusing on the application of nanotechnology in diagnostics, therapeutics, and drug delivery systems throughout the biomedical field. This journal is indexed on PubMed Central, MedLine, CAS, SciSearch $\AA$, Current Contents ${ }^{\circledR} /$ Clinical Medicine,

\section{Dovepress}

Journal Citation Reports/Science Edition, EMBase, Scopus and the Elsevier Bibliographic databases. The manuscript management system is completely online and includes a very quick and fair peer-review system, which is all easy to use. Visit http://www.dovepress.com/ testimonials.php to read real quotes from published authors. 